\title{
REFLEXIÓN SOBRE LA EVALUACIÓN EN LA FORMACIÓN UNIVERSITARIA
}

Ingrid Kaiser Aranda

Isabel Parés Gutiérrez

Mónica Villareal

\section{RESUMEN}

Cara al encuentro del alumno con el ámbito profesional, la evaluación universitaria cobra una importancia radical en el Proceso Enseñanza-Aprendizaje. Pero no puede tratarse, de ninguna manera, de una simple verificación numérica de lo que el profesor ha buscado transmitir.

Una verdadera evaluación -en cualquier grado, pero aún más en el universitario- requiere tener como objetivo valorar la auténtica formación de cada estudiante. Es por ello que la evaluación buscará ser continua, integral, sistemática... Se trata, entonces, de un instrumento flexible que proporciona un conocimiento profundo del alumno y que, gracias a ello, permite ayudarlo para mejorar su Proceso Enseñanza-Aprendizaje.

Vista así, la evaluación es un termómetro creativo que utiliza el docente comprometido en la mejora del alumno, de sí mismo y de la sociedad.

\section{INTRODUCCIÓN}

La evaluación es uno de los momentos esenciales que conforman el Proceso Enseñanza-Aprendizaje, y por ello, merece tener una sistematización y fundamentación, además de técnica, axiológica y ética que sustente la validez del propio proceso evaluativo; principalmente, porque se «evalúa para el alumno, para darle una información sobre su aprendizaje; se evalúa para el profesor, para que conozca los resultados de 
su acción; y se evalúa para el sistema, para certificar los resultados de los alumnos" ${ }^{1}$ y efectuar los ajustes pertinentes.

Actualmente, la evaluación está centrada en la mera acreditación de conocimientos propios del área de estudio que, en muchos casos, son exclusivamente memorísticos. Por ello, la evaluación condiciona de tal manera el proceso de enseñanza aprendizaje que bien podría decirse que el momento que preocupa al estudiante universitario no es el de su aprendizaje, sino el de su evaluación ${ }^{2}$.

Es frecuente encontrar enfoques que ven, en la evaluación, el final del proceso de formación, limitándola al plano de la medición de resultados; la evaluación abarca mucho más que la mera medición y acreditación ${ }^{3}$.

\section{Modelo tradicional de evaluación}

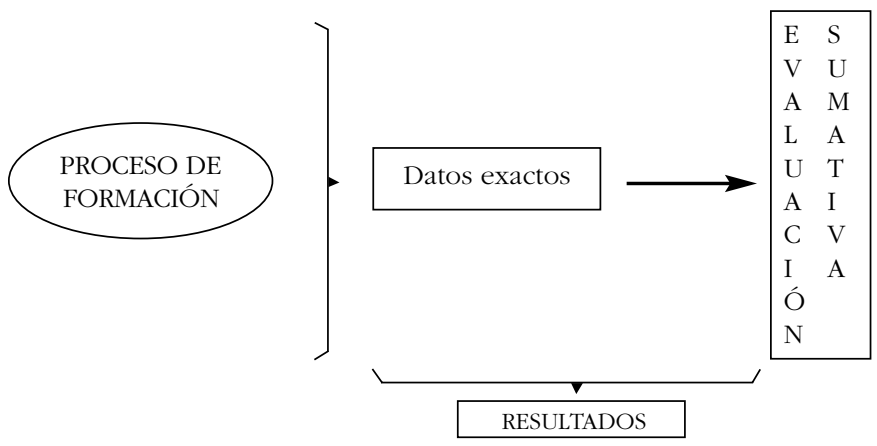

El supuesto objetivo de todo docente universitario hacia sus alumnos es la aprehensión de una serie de competencias que cumplan con los objetivos propios de la asignatura que imparte, con el fin de apoyar la formación del estudiante universitario para la vida laboral.

${ }^{1}$ SANTOS GUERRA, M. A., Evaluación educativa 2., p. 24.

${ }^{2}$ Cfr. ibidem., p. 25 .

3 "Medir es la acción de recoger información sobre el aprendizaje a través de un conjunto de técnicas (exámenes, pruebas, controles, etcétera), centrándose en el aspecto cuantitativo numérico". DOMÉNECH, F., Proceso de enseñanza aprendizaje., p.134. 
Para lograr esto se sugiere una ruptura de esquemas que aborde el Proceso Enseñanza-Aprendizaje desde una perspectiva integral. "De ahí que una modificación del concepto de evaluación debe partir necesariamente de una modificación en la naturaleza de la enseñanza" ${ }^{4}$.

Así, el reto del docente universitario inicia desde el tipo de enseñanza elegido, pues con esto construirá el contenido necesario a evaluar. "Es decir, que estudiar la evaluación es entrar en el análisis de la Pedagogía que es práctica y reflexionar sobre todos los elementos que la componen con el fin de sensibilizar a los profesores antes que preocuparnos de proporcionar modelos o aconsejar técnicas de evaluación concretas" ${ }^{5}$. Al asumir esta idea podremos iniciar con el tema que nos ocupa: la evaluación universitaria.

Se entiende por evaluación el proceso mediante el cual tanto el alumno como el docente obtienen una serie de elementos que les permitan ajustar y adecuar el estilo de enseñanza y las estrategias de aprendizaje; es decir, la evaluación busca realizar una valoración de la situación enseñanza aprendizaje sin enfocarse, necesariamente, a uno solo de los elementos que la componen.

Proceso continuo, integral y sistemático que busca valorar la eficacia y la eficiencia de la situación de enseñanza-aprendizaje.

Por lo que cabe "considerar la evaluación como el motor del cambio, como la rueda que pone en movimiento toda la maquinaria de la mejora del profesor, de la clase y de la escuela ${ }^{6}$.

\footnotetext{
${ }^{4}$ MORÁN OVIEDO, P., La docencia como actividad profesional., p.74. ${ }^{5}$ MARTÍN, M.L., Planeación, administración y evaluación de la educación., Trillas., México., 1997., p.180.

${ }^{6}$ DE VICENTE RODRÍGUEZ, P., Desarrollo profesional del docente: un modelo colaborativo de evaluación., p.114.
} 
Si una institución de Educación Superior desea elevar su calidad académica es necesario que ayude a los profesores para que realicen una evaluación integral del Proceso Enseñanza-Aprendizaje.

"La finalidad de la evaluación educativa es la de orientar y ayudar a tomar decisiones relativas al objeto de valoración" ${ }^{7}$. Por tal motivo, la evaluación está enfocada a la ayuda y al servicio de la formación del alumno; en esta búsqueda, la evaluación podrá abarcar lo siguiente: una evaluación continua, sistemática e integral.

\section{CARACTERÍSTICAS DE LA EVALUACIÓN UNIVERSITARIA}

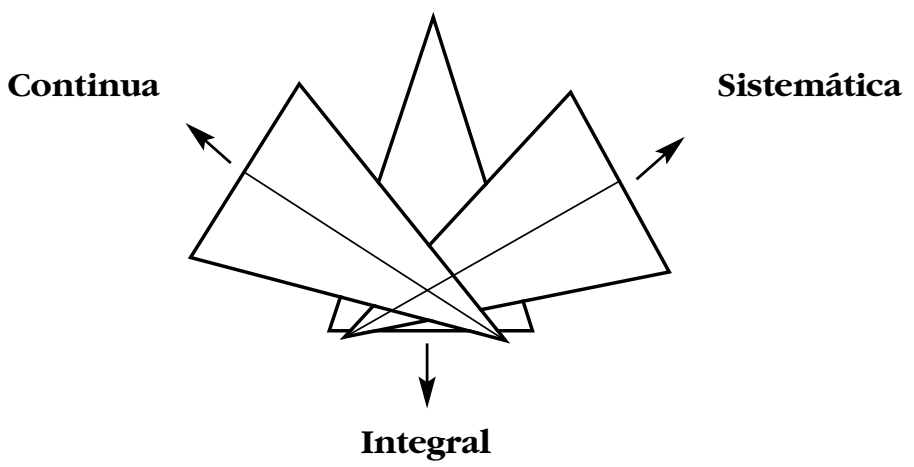

\section{Evaluación Continua}

Como su nombre lo indica, una vez iniciado el proceso de evaluación, no debe detenerse. Esto se refiere a implementar la evaluación en todos y cada uno de los momentos de enseñanza y de aprendizaje. Por lo tanto, la evaluación continua implica considerar tres momentos esenciales, sin importar el modelo que la sustente:

a) Evaluación inicial o diagnóstica.

b) Evaluación procesual o formativa.

c) Evaluación final o sumativa.

${ }^{7}$ DOMÉNECH, F. op.cit., p.134. 


\section{Evaluación Continua}

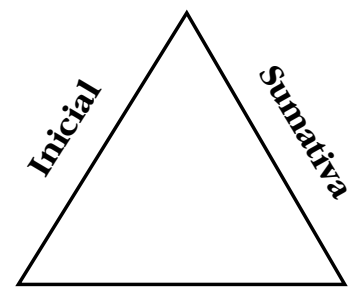

Final

\section{Evaluación inicial}

La evaluación inicial es aquella que busca conocer el estado actual de una situación educativa; se preocupa por establecer indicadores que le permitan reflejar, mediante el empleo de diversos instrumentos que evalúen, el estado del arte de los conocimientos, habilidades y actitudes del educando, en este caso universitario.

Tiene como finalidad esencial conocer al alumno al que se va a formar. Es, en palabras de García Hoz, "conocer para educar, cuya justificación se halla en otro principio de parecido sonido y grafismo: conocer para adecuar; adecuar el diseño, el proyecto educativo a cada persona como consecuencia de su individualidad" ${ }^{8}$.

Por esto, resulta ideal llevar a cabo este proceso antes del diseño de la planeación educativa y al momento de establecer contacto con el alumno; esto dará resultados tangibles que favorezcan la calidad académica de una institución de Educación Superior.

\section{Evaluación formativa}

En segundo término se encuentra la evaluación formativa que se «encarga de valorar cada uno de los aspectos procesuales de cualquier tarea" ".

${ }^{8}$ GARCÍA HOZ, V., Diagnóstico, evaluación y toma de decisiones., p.49.

${ }^{9}$ Enciclopedia de Pedagogía., Tomo V., p.1047. 
En pocas palabras es la evaluación de los procesos posteriores a la etapa diagnóstica y previos a los resultados consecuentes de dichos procesos educativos.

El término de evaluación formativa fue acuñado por M. Scriven en 1967. Afirma que ésta «incluye en su definición la necesidad de valorar el objeto evaluado, es decir, de señalar lo positivo o negativo, el mérito o demérito de lo conseguido (...) supone añadir nuevos elementos al proceso evaluativo, la ideología del evaluador y el sistema de valores de la sociedad, que condicionarán los resultados de cualquier estudio si no se adoptan los procedimientos y las medidas correctivas para evitar los sesgos posibles" ${ }^{10}$.

Esto implica evaluar, ponderando los aspectos favorables y señalando los contrarios, para poder reformular las estrategias metodológicas del proceso educativo.

Resulta muy recomendable el uso del portafolio o portfolio ${ }^{11}$ para poder observar, de manera objetiva, el desarrollo del proceso mismo.

\section{Evaluación sumativa}

En último término encontramos la evaluación sumativa también conocida como final. Frecuentemente se estudian autores que definen la evaluación como un análisis del alcance que el alumno tuvo con respecto a los objetivos propuestos. Esta visión limita la trayectoria de la evaluación al tercer momento del modelo.

Es precisamente la evaluación sumativa la que se realiza al término de los procesos, valora los resultados del aprendizaje.

${ }^{10}$ Enciclopedia de Pedagogía., Tomo III., p.618.

${ }^{11}$ Se entiende por portafolio el compendio de trabajos elaborados por el alumno que permiten reflejar cómo se ha desarrollado éste a lo largo de una asignatura, de un ciclo escolar o de una etapa determinada. Es muy usado para: certificación basada en procesos de aprendizaje; reflexión sobre el propio conocimiento del alumno; comunicación de la situación actual con padres, compañeros, profesores, entre otros; así como para centrar el proceso educativo en el alumno, en su propio aprendizaje. 
Al igual que la evaluación formativa, se emplea habitualmente para procesos de acreditación escolar y certificación académica.

Estos tres momentos que componen la evaluación continua, deben observarse de manera conjunta; si sólo se enfoca el proceso de evaluación a uno o dos de ellos, será imposible hablar de una evaluación continua. Además, para lograrla, es necesario que se continúe ininterrumpidamente el proceso.

Seguir un modelo de evaluación continua colabora a la mejora constante del sistema escolar, de los procesos de enseñanza del docente universitario y de los procesos de aprendizaje del alumno, mediante una continua retroinformación.

Esta evaluación es parte de un modelo integral que permite tomar decisiones oportunas que favorezcan la adecuación del contenido a la innovación y mejora social del entorno. "La continuidad de la evaluación es una de las condiciones básicas del éxito en la acción educativa, ya que permite constatar las dificultades, los errores y las lagunas apenas se producen y no cuando se han afianzado" ${ }^{12}$.

Todo docente universitario que desee ser competente y formar alumnos competitivos debe, necesariamente, formular un modelo de evaluación continua que se adapte, además de al modelo escolar de la institución, a su contexto y circunstancias particulares.

El modelo de evaluación continua permite conocer las particularidades propias de cada alumno, con la finalidad de establecer objetivos y estrategias personalizadas y adecuadas para el desarrollo integral de ese educando.

${ }^{12}$ GARCÍA HOZ, V. Diagnóstico, evaluación y toma de decisiones., p.46. 


\section{EVALUACIÓN INTEGRAL}

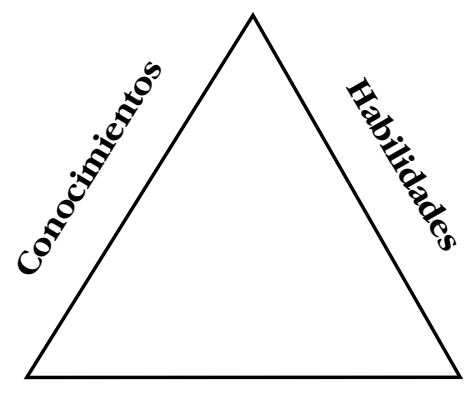

Actitudes

La evaluación debe ser integral desde dos puntos de vista:

- Con respecto al alumno.

- Con respecto al Proceso Enseñanza-Aprendizaje.

\section{Con Respecto al Alumno}

Deben evaluarse conocimientos, habilidades y actitudes. La evaluación de los contenidos requiere ser distinta en cada asignatura, pues tiene que estar acorde al tipo de contenido que se quiera evaluar.

\section{a) Contenidos conceptuales}

Los contenidos conceptuales deben evaluarse de acuerdo al grado o nivel de profundización que se quiera alcanzar.

Las actividades propias de la evaluación para este tipo de contenidos son:

- Observación del uso del concepto en diversas situaciones.

- Debates.

- Diálogos.

- Resolución de problemas. 


\section{b) Contenidos factuales}

Los contenidos factuales se refieren a los hechos. Para evaluarlos, se necesita una asociación de conceptos. Las actividades de evaluación de contenidos factuales aluden a la utilización conjunta de hechos y conceptos.

Los aspectos a tomar en cuenta para evaluar este tipo de contenidos son:

- Rapidez.

- Alterar la secuencia de lo visto en clase.

\section{c) Contenidos procedimentales}

Los contenidos procedimentales se refieren al saber hacer, es decir, al procedural knowledge". La evaluación de los contenidos procedimentales consiste en verificar el dominio de la habilidad en la práctica. Se evalúa a través de la observación sistemática en actividades hechas en clase.

\section{d) Contenidos actitudinales}

La evaluación de los contenidos actitudinales está compuesta por elementos cognitivos, conductuales y afectivos. Por lo tanto su evaluación es muy compleja.

Los problemas entorno a este aspecto son:

- La valoración del profesor puede ser subjetiva, debido a sus posiciones ideológicas.

- Existe poca tradición práctica en la evaluación de actitudes, por lo que se cuestiona la necesidad de evaluarlos.

Cómo realizar la evaluación de este tipo de contenidos:

- Observar el comportamiento de los alumnos en forma sistemática.

- Observar el comportamiento de los alumnos en diferentes situaciones grupales.

Cabe resaltar que hay que aprovechar las situaciones difíciles y los momentos de conflicto para evaluar las actitudes. 


\section{Con Respecto al Proceso Enseñanza-Aprendizaje}

Se debe evaluar toda la instrumentación didáctica, es decir los elementos y los momentos didácticos, ya que la eficacia y la eficiencia del Proceso Enseñanza-Aprendizaje se debe a todo un proceso integral.

Se mencionarán algunos aspectos a evaluar en torno a los elementos y momentos didácticos:

\begin{tabular}{|c|c|}
\hline ELEMENTOS DIDÁCTICOS & ASPECTOS A EVALUARSE \\
\hline Educando & $\begin{array}{l}\text { - Conocimientos. } \\
\text { - Habilidades. } \\
\text { - Actitudes. }\end{array}$ \\
\hline Educador & $\begin{array}{l}\text { - Preparación. } \\
\text { - Gusto por impartir la materia. } \\
\text { - Actualización. } \\
\text { - Seguimiento del proceso } \\
\text { de aprendizaje de los alumnos. } \\
\text { - Reflexionar en torno a } \\
\text { su papel como docente. } \\
\text { - Habilidades docentes. } \\
\text { - Habilidades didácticas. }\end{array}$ \\
\hline Objetivos educativos & $\begin{array}{l}\text { - Cumplimiento. } \\
\text { - Razones por las que se } \\
\text { cumplieron o no. }\end{array}$ \\
\hline Contenidos educativos & $\begin{array}{l}\text { - Pertinencia. } \\
\text { - Secuencia lógica. } \\
\text { - Adaptabilidad al educando. }\end{array}$ \\
\hline Estrategias didácticas & $\begin{array}{l}\text { - Adecuación. } \\
\text { - Funcionamiento. } \\
\text { - Relación con los objetivos y contenido }\end{array}$ \\
\hline Recursos didácticos & - Medio. \\
\hline Tiempo didáctico & $\begin{array}{l}\text { - El necesario, acorde a los objetivos. } \\
\text { - Control adecuado del tiempo. }\end{array}$ \\
\hline Lugar & $\begin{array}{l}\text { - Adecuación. } \\
\text { - Preparación. }\end{array}$ \\
\hline
\end{tabular}




\begin{tabular}{|l|l|}
\hline MOMENTOS DIDÁCTICOS & ASPECTOS A EVALUARSE \\
\hline Diagnóstico & - Elaboración. \\
& - Real. \\
& - Cumplimiento de funciones. \\
\hline Planeación & - Sistemática. \\
& - Global. \\
& - Real. \\
& - Clara. \\
\hline Realización & - Empuje. \\
& - Coordinación. \\
& - Manejo de contenidos. \\
& - Manejo de estrategias. \\
& - Manejo de recursos. \\
& $\begin{array}{l}\text { - Sanejo de imprevistos. } \\
\text { - Continua. }\end{array}$ \\
\hline Evaluación & Integral. \\
\hline
\end{tabular}

\section{EVALUACIÓN SISTEMÁTICA}

Se entiende por evaluación sistemática al tipo de evaluación que pretende valorar y dar seguimiento cercano, y un tanto crítico-reflexivo, al mismo proceso de formación, pues comprende la autoevaluación por parte del alumno, la evaluación de los mismos docentes, y la evaluación grupal.

Esto con la finalidad de evaluar con un sentido más global a la formación que se está brindando, de tal modo que el alumno tome realmente un papel activo y central dentro de dicho proceso, pues de esta forma percibirá la evaluación como una herramienta indispensable para su crecimiento, en vez de considerarla el principal obstáculo para la finalizar sus estudios. 


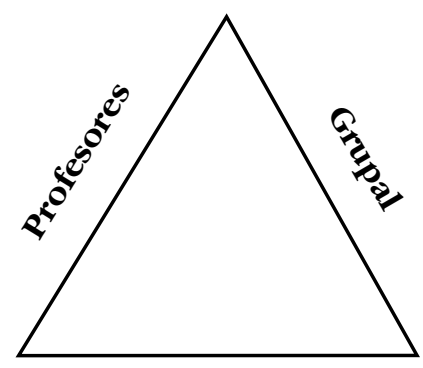

Autoevaluación

\section{Autoevaluación}

La autoevaluación será el tema principal de este apartado, puesto que el alumno debe considerar, dentro de su etapa formativa, una reflexión crítica y valorativa. Es a éste a quien le corresponde estar convencido de hacer sistemática y constantemente una valoración de sí mismo, porque entonces comprenderá y aceptará los otros tipos de evaluación. No obstante, "nos encontramos con que se concibe la autoevaluación de los alumnos como una trampa que facilita la consecución de buenas calificaciones (...)" ${ }^{13}$. De ahí que quien tiene la misión de encaminar al alumno hacia una auténtica reflexión crítica es el docente, facilitándole los lineamientos necesarios para la consecución de una autoevaluación objetiva.

Ahora bien, la autoevaluación merece iniciar con una primera reflexión; implica una mirada hacia el interior mediante la cual la persona analiza y aprehende el mapa general de lo que ella misma es.

Es posible decir que la reflexión es la operación indicada para tener una autoaprehensión que permitirá un auténtico autodominio. En la medida en la que se avanza en el conocimiento del yo, se tiene una mayor noción de los propios

${ }^{13}$ SANTOS GUERRA, M.A., op.cit., p. 35. 
alcances y reacciones, limitaciones y puntos de oportunidad; aspecto necesario para la formación de alumnos autodidactas, lo cual implica formar en y para la libertad.

En este sentido, el alumno debe hacer un tipo de evaluación comparativa en tanto que valore el estado en el que se encontraba anteriormente con el actual. Por lo que debe percatarse del nivel y tipo de cambios que surgieron en él y las futuras acciones y actitudes que necesita llevar a cabo para avanzar en el plano cognitivo, actitudinal y de habilidades. Así, el alumno podrá valorar "los efectos laterales, secundarios e imprevistos, siendo éstos en ocasiones mucho más importantes que los resultados buscados directamente (...) ${ }^{14}$.

Principalmente, con los estudiantes universitarios es preciso promover la autoevaluación, puesto que ello implica prepararlos auténticamente para la vida laboral. Se trata de formar profesionistas autodidactas que no esperen recibir una evaluación exterior para crecer tanto personal como profesionalmente, por lo que se proponen algunas estrategias para conseguirlo:

- La reflexión escrita en la cual se cuestione al alumno sobre la aprehensión de los conocimientos mínimos que debe cubrir la materia.

- El desarrollo de ciertas habilidades indispensables para la aplicación de dichos conocimientos y la generación de actitudes propias de un estudiante universitario.

- Deberá obtenerse una evaluación cualitativa en donde fundamente la evaluación cuantitativa.

\section{Evaluación de los Docentes}

Este tipo de evaluación hace referencia a la valoración objetiva que debe realizar el profesor, la cual incluye la evaluación continua (inicial, formativa y final) y la evaluación integral (conocimientos, habilidades y actitudes).

${ }^{14}$ MARTÍN, M.L., op.cit., p. 181. 
Cabe mencionar que el papel del docente, como actor evaluador, merece gran importancia pues debe evitar ciertos vicios que se cometen constantemente en la práctica, tales como ${ }^{15}$ :

- Evaluar sólo al alumno (sin considerar el currículo y el propio sistema), los resultados, o los conocimientos.

- Enfocarse exclusivamente al aspecto negativo.

- No considerar el contexto ni adecuar los instrumentos.

- Centrarse únicamente en la evaluación cuantitativa.

- No realizar un proceso de evaluación acorde al propio proceso de enseñanza aprendizaje que se ha llevado a cabo.

- Evaluar con esquemas repetidos (sin ajustar de acuerdo a los cambios que se van gestando).

- Utilizar la evaluación como un medio controlador.

- No realizarla continuamente (lo cual consiste en recoger información en todo momento).

- Dar por hecho que el alumno comprende el sistema de evaluación.

- No considerar la idea de evaluar la misma evaluación.

La formación universitaria debe considerar un sistema de evaluación totalizador. Porque a través de la evaluación, el profesor conoce los procesos que han llevado a cabo sus alumnos, lo cual le permitirá ajustar su modelo de enseñanza, y por ende, profundizar sobre su propia labor. Se propone la aplicación de algunas herramientas de evaluación adecuándolas según el contenido de la materia. Es recomendable que durante un semestre se apliquen la mayoría para dar dinamismo al sistema de evaluación:

- Exámenes escritos (con preguntas abiertas para desarrollar la redacción, capacidad de análisis y fundamentación, y con preguntas cerradas para afianzar la aprehensión de conceptos, estimular la memorización necesaria para ciertos contenidos y desarrollar la capacidad de síntesis).

${ }^{15}$ Cfr. MARTÍN, M.L., op.cit., pp.180-184. 
- Exámenes orales (para fomentar el desarrollo de la argumentación oral).

- Estudio de casos (para argumentar la solución de situaciones reales que se gestan en la vida laboral y aplicar los conocimientos de la materia).

- Trabajos de investigación.

- Portafolio.

- Análisis de lecturas.

- Creación de proyectos individuales o en equipo.

- Prácticas profesionales.

En la actualidad, la formación universitaria aún tiene un lenguaje numérico que indica quién ha aprobado o no, aspecto que debe enriquecerse de tal forma que ese número asignado sea el reflejo de todo un modelo de evaluación continuo, integral y sistémico.

\section{Evaluación Grupal}

La evaluación grupal debe formar parte del proceso de evaluación sistemática, pues comprende la valoración en conjunto que la comunidad hace de sí misma. Dicha práctica hace consciente, tanto al alumno como al profesor, de la incidencia inevitable que se tiene en los otros, por tanto, el compromiso de mejora no es sólo hacia uno mismo sino también hacia el grupo. Esta situación involucra a todas las partes para ir caminando sobre una misma línea que facilita, indudablemente, el proceso de enseñanza aprendizaje.

Asimismo, «la evaluación es también una tarea que ayuda a la revisión del proceso grupal, en términos de las condiciones en que se desarrolló, los aprendizajes alcanzados, los no alcanzados, así como las causas que posibilitaron o imposibilitaron las consecución de metas propuestas" ${ }^{16}$. La evaluación requiere de una visión global, en cuanto al grupo, y particular,

${ }^{16}$ MORÁN OVIEDO, P., op.cit., p.72. 
en cuanto a las personas. Ello con la finalidad de obtener la máxima información para retroalimentar, objetivamente, tanto al educando como al docente y al mismo sistema de enseñanza aprendizaje. Este tipo de evaluación puede llevarse a cabo a través de:

- Mesa panel.

- Diálogo abierto.

- Debate.

- Evaluación escrita, tanto cuantitativa como cualitativa, por parte de cada uno de los integrantes del grupo.

Continua:

¿cuándo?
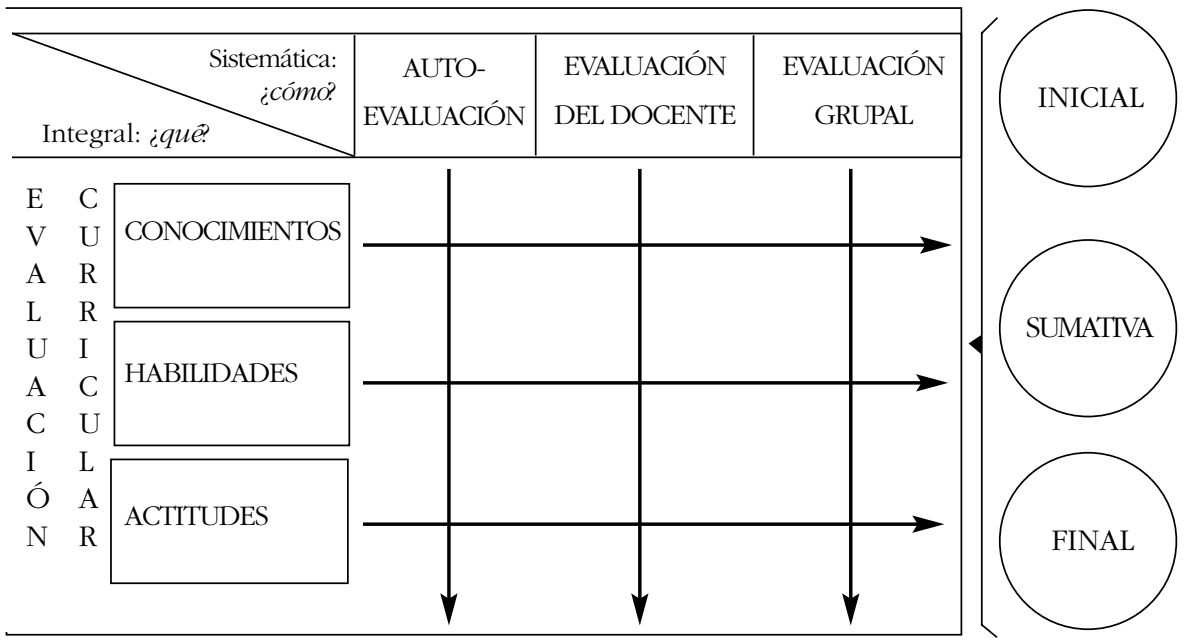

\section{CONCLUSIÓN}

A continuación se presentan algunas frases para la reflexión, basadas en lo expuesto a lo largo de este artículo:

- La formación permanente requiere evaluación permanente.

- Hay tantos aspectos a evaluar que el docente universitario debe realizar una evaluación colegiada que elimine, al máximo, los sesgos subjetivos del profesor. 
- El modelo evaluador debe estar acorde al modelo de enseñanza/aprendizaje.

- Es esencial, en la evaluación, la información arrojada y cómo es utilizada (retroalimentación).

- Detectar los puntos fuertes y las áreas de oportunidad en el Proceso Enseñanza-Aprendizaje.

- Disponer una solución en las áreas de oportunidad.

- Verificar si la intencionalidad se logró y analizar sus causas.

- Aprender de la experiencia: continuar con lo positivo y reelaborar estrategias para los puntos de mejora.

- Proporcionar un conocimiento profundo del alumno que permite ayudarlo para mejorar su Proceso EnseñanzaAprendizaje.

- La evaluación es una parte del Proceso EnseñanzaAprendizaje que siempre debe estar presente. 
DE VICENTE RODRÍGUEZ, P., Desarrollo profesional del docente: un modelo colaborativo de evaluación., I.C.E.Universidad de Deusto., Bilbao., 2002., 358 p.

DOMENECH, F., Proceso de enseñanza aprendizaje., Universitat Jaume Primera., Madrid., España., 1999., 175 p.

KNOX, A., Evaluation for continuing education., Jossey-Bass., Estados Unidos de América., 2002., 334 p.

Enciclopedia de Pedagogía., Editorial Espasa., España., 2002., 5 Tomos., 1115 p.

GARCÍA HOZ, V., Diagnóstico, evaluación y toma de decisiones., Rialp., Madrid., 1989., 423 p.

MARTÍN, M.L., Planeación, administración y evaluación de la educación., Trillas., México., 1997., 230 p.

MORÁN OVIEDO, P., La docencia como actividad profesional., Gernika., México., 1997., 189 p.

SANTOS GUERRA, M.A., Evaluación educativa 2., Magisterio del Río de la Plata., Argentina., 1996., 270 p. 